INPLASY

PROTOCOL

To cite: Hu et al. Effects of inspiratory muscle training on coronary artery bypass graft surgery: a protocol of systematic review and metaanalysis. Inplasy protocol 2021120089. doi: 10.37766/inplasy2021.12.0089

Received: 20 December 2021

Published: 20 December 2021

Corresponding author: Yu Hu

myhuyu_0503@163.com

Author Affiliation:

1. The Third People's Hospital of Chengdu/Affiliated Hospital of Southwest Jiaotong University 2. West China Hospital

Support: Yes.

Review Stage at time of this submission: Preliminary searches.

Conflicts of interest: None declared.

\section{Effects of inspiratory muscle training on coronary artery bypass graft surgery: a protocol of systematic review and meta-analysis}

\author{
Hu, Y1; He, L2; Wang, YC³; Luo, XZ"; Zhao, X5; Meng, LJ6.
}

Review question / Objective: The aim of our study is to investigate the effects of inspiratory muscle training on patients undergoing coronary artery bypass graft (CABG) surgery.

Condition being studied: Decreased inspiratory muscle strength, exercise capacity, quality of life and pulmonary function and the risk of pulmonary complications in coronary artery bypass graft.

Eligibility criteria: Inclusion criteria: 1. Participants undergoing CABG, regardless of gender, age, race, nationality, and other characteristics; 2 . the intervention was inspiratory muscle training; 3. therapy in the comparison groups can be sham placebo, pharmacotherapy or no any additional intervention to usual care. Exclusion criteria: 1. RCTs with crossover design; 2; inspiratory muscle training combined with other active methods except for medication for CABG; 3; full texts cannot be found; 4. duplicate or insufficient data; 5. Case report; 6. animal experiment.RCTs with any of the following conditions will be excluded.

INPLASY registration number: This protocol was registered with the International Platform of Registered Systematic Review and Meta-Analysis Protocols (INPLASY) on 20 December 2021 and was last updated on 20 December 2021 (registration number INPLASY2021120089).

\section{INTRODUCTION}

Review question / Objective: The aim of our study is to investigate the effects of inspiratory muscle training on patients undergoing coronary artery bypass graft (CABG) surgery.

Condition being studied: Decreased inspiratory muscle strength, exercise capacity, quality of life and pulmonary 
function and the risk of pulmonary complications in coronary artery bypass graft.

\section{METHODS}

Participant or population: All patients had confirmed diagnosis of coronary artery disease and were scheduled to undergo coronary artery bypass graft (CABG) surgery. No restrictions will be set on gender, race or nation.

Intervention: Inspiratory muscle training.

Comparator: Alternative active treatment or no treatment.

Study designs to be included: Only studies classified into randomized controlled trials (RCTs) will be included.

Eligibility criteria: Inclusion criteria: 1. Participants undergoing CABG, regardless of gender, age, race, nationality, and other characteristics; 2 . the intervention was inspiratory muscle training; 3 . therapy in the comparison groups can be sham placebo, pharmacotherapy or no any additional intervention to usual care. Exclusion criteria: 1. RCTs with crossover design; 2; inspiratory muscle training combined with other active methods except for medication for CABG; 3; full texts cannot be found; 4 . duplicate or insufficient data; 5. Case report; 6. animal experiment.RCTs with any of the following conditions will be excluded.

Information sources: Sources will be searched in PubMed, PEDro, the Cochrane Library, Embase, China Biology Medicine (CBM), China National Knowledge infrastructure (CNKI), Wan Fang Data and the Chinese Science and Technology Periodical Database (VIP).

Main outcome(s): Outcomes include inspiratory muscle strength (Maximum inspiratory pressure), pulmonary function (FEV1, FVC, FEV1/FVC), 6-min walk test, exercise capacity (PeakVO2), cardiopulmonary exercise test variables
(RER, VE, VE/VCO2) and quality of life questionnaire (HADS).

Quality assessment / Risk of bias analysis: Two reviewers will independently assess the methodological quality of the included RCTs based on Cochrane risk-of-bias criteria. Each item will be rated as low risk, high risk, or unclear risk. Discrepancy will be solved by discussion.

Strategy of data synthesis: We will perform meta-analysis via Stata software (version 15.1) and $R$ software (version 4.1.0). Standardized mean difference (SMD) will be used for continuous outcomes and relative ratio (RR) will be used for dichotomous outcomes. The uncertainty will be presented with $95 \%$ confidence intervals (95\% Cls). Heterogeneity will be explored by using a standard $X^{2}$ test with a significance level of $P<0.10$. The $I^{2}$ statistic will be used to quantify inconsistency. If $P>0.1$ and $I^{2}<50 \%$, fixed effect model will be used due to acceptable heterogeneity. If $P<0.1$ and/or $I^{2} \geq 50 \%$, random effect model will be used since statistical heterogeneity may be exist.

Subgroup analysis: To investigate potential heterogeneity across studies, we will further explore heterogeneity by subgroup analysis and meta regression based on the following factors: age, gender, types of intervention and living conditions.

Sensitivity analysis: We will conduct sensitivity analysis according to sample size, study design, heterogeneity quality, methodological quality and statistical methods, exclude the low-quality trials and ensure the stability of analysis results.

Country(ies) involved: China.

Keywords: coronary artery bypass graft (CABG), inspiratory muscle training, quality of life, exercise capacity, pulmonary function.

Contributions of each author:

Author 1 - YU HU.

Author 2 - LIN HE. 
Author 3 - YUCHAN WANG.

Author 4 - XIAOZHOU LUO.

Author 5 - XIN ZHAO.

Author 6 - LIJIAO MENG. 